\title{
Outcome of complete acellular dermal matrix wrap with polyurethane implant in immediate prepectoral breast reconstruction
}

\author{
Foteini Neamonitou, Senthurun Mylvaganam, Fathi Salem, Raghavan Vidya \\ Oncoplastic Breast, Department of General Surgery, The Royal of Wolverhampton NHS Trust, Wolverhampton, UK
}

Background Polyurethane implants have been used on and off in breast reconstruction since 1991 while prepectoral breast reconstruction has gained popularity in recent times. In this study, we present our outcomes from the use of acellular dermal matrix (ADM) complete wrap with polyurethane implants in prepectoral breast reconstruction.

Methods This is a retrospective review of prospectively maintained database from 41 patients receiving complete ADM wrap with prepectoral polyurethane implants over a 3-year period. Selection criteria were adapted from a previous study (4135 Trust Clinical Audit Database) evaluating prepectoral reconstruction with Braxon matrices. Patient demographics, operative data, surgical complications, and outcomes were collected and analyzed.

Results A total of 52 implant reconstructions were performed in 41 patients with a mean follow-up of 14.3 months (range, 6-36 months). The overall reported complication rates including early (less than 6 weeks) and late complications. Early complications included two patients (4.9\%) with wound dehiscence. One of which had an implant loss that was salvageable. Another patient (2\%) developed red-breast syndrome and two women (4.9\%) developed with seroma treated conservatively. Late complications included one patient (2\%) with grade II capsular contraction, 12 patients with grade I-II rippling and two patients (4.9\%) with grade III rippling.

Conclusions We present our experience of prepectoral polyurethane implant using complete ADM wrap. This is one of the few papers to report on the outcome of the prepectoral use of polyurethane in immediate implant-based breast reconstruction. Our early observational series show satisfactory outcome and long-term results are warranted by a large multicenter study.

Keywords Polyurethane / Implant capsular contracture / Breast implants / Acellular dermal matrix
Correspondence: Foteini Neamonitou Oncoplastic Breast, Department of General Surgery, The Royal of Wolverhampton NHS Trust, Wolverhampton Road, Wolverhampton, West Midlands WV10 0QP, UK

Tel: +44-1902-307999

Fax: +44-1902-695600

E-mail: foteini.neamonitou@nhs.net

Received: June 26, $2020 \bullet$ Revised: September 29, $2020 \bullet$ Accepted: October 6, 2020

pISSN: 2234-6163 • elSSN: 2234-6171・https://doi.org/10.5999/aps.2020.01207• Arch Plast Surg 2020;47:567-573

\section{INTRODUCTION}

The use of silicone and or saline-based breast implants has been widespread for a number of years, particularly in the augmenta- tion setting. However, new technologies and ever-evolving use and research have led to changing practices in a variety of settings, especially in breast reconstruction following surgery for breast cancer which brings with it unique considerations [1]. 
While the development of capsular contracture is a well-recognized potential complication of breast implants, this is exacerbated by the use of postmastectomy radiotherapy [2]. Various attempts have been made to try and reduce this effect, but the rates remain higher than in nonirradiated breast implants [3].

One such technique, which has offered a protective benefit against capsular contracture, is the coating of implants with polyurethane foam. It is proposed that the irregular interconnecting coating on the implant surface and associated foreign body reaction prevents the formation of a single longitudinal capsule. The irregular capsule formed allows for a more distributed tension, reducing the occurrence of a circumferential contracture.

Polyurethane foam coated breast implants were first introduced in the 1970s [4]. Their widespread use was prevented when the Food and Drug Administration retracted their approval in 1991 due to the potential carcinogenic nature of 2,4-toluenediamine (TDA), the breakdown product of the polyurethane coating [5]. However, the risk at that time was not felt to be significant enough to warrant removal of those implants that had already been used [6]. In 1991 Surgitek polyurethane coated breast implants were voluntarily removed from the market in Europe as well [7].

Subsequent reviews of those patients who had already received these implants, as well as animal studies, demonstrated that the risk of 2,4 TDA related cancer was smaller than previously believed. The current belief is that the risk is approximately 1 in 1.1 million. The current risk of implant-associated anaplastic largecell lymphoma is 1 in 30,000 with some variability based on implant choice. Based on a review of the safety data CE (Conformité Européene) approval was granted in 2006 in the United Kingdom for the use of polyurethane foam coated breast implants [5].

Implant-based breast reconstruction has continued to evolve as interventions and techniques are introduced to address complications such as capsular contracture, improve postoperative recovery and aesthetic outcome. The most recent strategy to be seen is the use of prepectoral placement of breast implants. Whilst not entirely novel, the rapidly spreading availability of acellular dermal matrices (ADM) has allowed this technique to be used with much more confidence, particularly addressing the concern over controlling the implant pocket. They may also act to reduce palpable rippling of the implant surface in particular in the presence of thin mastectomy skin flaps. A clear benefit of this technique is the decrease in morbidity produced by avoiding disrupting the pectoralis major muscle, which otherwise would be dissected to create part of the implant pocket [8].

Literature is scant on the use of complete ADM wrap using polyurethane implant and we present our initial experience using this technique.

\section{METHODS}

\section{Study setting and ethical consideration}

This is a retrospective review of prospectively maintained database of 41 patients, who were consented for publication of their data. These patients received breast reconstruction with prepectoral polyurethane implants from February 2015 until December 2019. The patients were followed-up until today with mean follow-up of 14.3 months and range 6 to 36 months including their outcome and complications as well.

Selection criteria were adapted from a previous study evaluating prepectoral reconstruction with Braxon matrices [9] and the study was approved by the local audit department (audit No. 4135).

Patient demographics, operative data, surgical early (less than 6 months) and late complications (more than 6 months) along with outcomes were collected and analyzed.

\section{Surgical technique \\ Preoperative setting}

All patients received a stat dose of antibiotics at induction, and postoperative antibiotics are administered after risk stratification. The technique of prepectoral implant breast reconstruction using complete $\mathrm{ADM}$ wrap using Braxon mesh has been described previously [10].

\section{Intraoperative setting}

Prepectoral implant-based reconstruction with polyurethane was performed as single-stage reconstruction of the breast. The mastectomy flaps were raised after carrying out dissection in the normal oncoplastic plane [10]. The dissection was carried out using diathermy at low settings. It was ensured that the mastectomy flaps had good viability and care was taken to preserve the perforators [11].

The Braxon preshaped mesh is a porcine derived ADM was used. The polyurethane implant is covered by the Braxon preshaped mesh ex vivo secured with absorbable sutures (2-0 Vicryl) to form a snug pocket. The mesh is placed over the chest wall and the unit is secured using three cardinal sutures at 12,3 , 9 o'clock position with 2-0 Vicryl suture.

The anterior implant pocket utilizes alternatives to the pectoralis major muscle to achieve complete anterior coverage. The posterior coverage was formed by the underlying pectoralis major muscle and the pectoralis fascia was preserved (where possible) to minimize postoperative pain [12]. 
Important to mention is that in large ptotic breasts, a dermal flap with use of an ADM/mesh was employed [13]. The presence of a dermal flap contributes to lower pole soft tissue coverage while the $\mathrm{ADM} /$ mesh completes the coverage superiorly as necessary [14]. These techniques allow for surgeon control of the implant pocket and may also implant visibility, particularly at the upper pole.

After the fixation of the implant, closed suction drains were used tunneled well away from the implant pocket. The drains were usually removed when the drainage is minimal over 24 hours varying from 10 to $30 \mathrm{~mL} /$ day. In our experience, this is usually 5-10 days post-surgery [15].

\section{RESULTS}

A total of 41 patients underwent an immediate prepectoral breast reconstruction using $\mathrm{ADM}$ complete mesh wrap (Braxon) and polyurethane implants in 52 breasts between Jan 2016 to December 2019 with a mean follow-up of 14.3 months (range, 6-36 months). Patient characteristics are shown in Table 1. The average age was $53.9 \pm 9.7$ years (range, $31-74$ years) and the average body mass index was $29.5 \pm 4.4 \mathrm{~kg} / \mathrm{m}^{2}$ (range, 19.4$37.0 \mathrm{~kg} / \mathrm{m}^{2}$ ). Four patients were smokers (9\%), one insulin-de-

\section{Table 1. Demographic data}

\begin{tabular}{|lc|}
\hline Characteristics & Data (\%) \\
\hline No. of patients & 41 \\
No. of breasts & 52 \\
Age (yr) & \\
Mean \pm SD & $53.9 \pm 9.7$ \\
Range & $31-74$ \\
BMI (kg/m²) & \\
Mean \pm SD & $29.5 \pm 4.4$ \\
Range & $19.4-37.0$ \\
Smoking & $4(9)$ \\
Diabetes & \\
IDDM & $1(2)$ \\
NIDDM & $2(5)$ \\
Radiotherapy & \\
Preoperative & $5(12)$ \\
Postoperative & $12(29)$ \\
Breast reconstructions & \\
Unilateral & $30(73)$ \\
Bilateral & $11(27)$ \\
Size of the implant (g) & \\
Mean \pm SD & $438.15 \pm 134.14$ \\
Range & $85-615$ \\
Follow-up time (mon) & \\
Mean \pm SD & 14.3 \\
Range & $6-36$ \\
\hline BMl, body mass index; IDDM, insulin-dependent diabetes mellitus; NIDDM, non- \\
IDDM. \\
\hline
\end{tabular}

pendent diabetes mellitus (IDDM; 2\%), and two non-IDDM (5\%). The mean volume of the mastectomies was $576.9 \pm 327.3$ $\mathrm{g}$ (range, 56-1,060 g).

Five of these patients $(12 \%)$ received preoperative radiotherapy, and 12 (29\%) received postoperative radiotherapy. Thirty of the population (50\%) received unilateral breast reconstructions, and the other eleven (50\%) received bilateral breast reconstructions. The mean size of the implants used was $438.15 \pm 134.14 \mathrm{~g}$ (range, 85-615 g).

The complications seen are detailed in Table 2 . There were no cases of postoperative hematoma, necrosis, infection, implant rotation during the follow-up period. Early complications included two patients (4.9\%), who developed both seroma and subsequent dehiscence, one patient (2\%) had implant loss which was salvaged by an expander implant and consequently received a fixed volume implant in 6 months after the implant loss. Important to mention is that the latter patient was obese and non-insulin dependent diabetic. She also had received two sessions of postoperative radiotherapy.

One patient (2\%) developed red-breast syndrome, delayedtype hypersensitivity response to the implant 4 months postoperatively, after the second session of postoperative radiotherapy.

Late complications included one patient (2\%) who received postoperative radiotherapy developed a grade II capsular contraction over 3 years follow-up. Furthermore, we offered lipomodelling in 14 patients (34\%) for better cosmesis and shaping of the breast of which four (9.7\%) of these patients received preoperative radiotherapy, and $10(24.3 \%)$ received postoperative radiotherapy. We observed grade I-II rippling in 12 patients and two patients had grade III rippling. Although patients were offered lipomodelling majority declined as it the rippling was mild (grade I-II) and only two patients (4.9\%) with significant rippling (grade III) underwent lipomodelling with satisfactory aesthetic outcome. The fat was harvested using tumescent technique using micro air kit. Approximately $130 \mathrm{~mL}$ of fat and 100

\section{Table 2. Complications}

\begin{tabular}{|lc|}
\hline Complications & No. of breasts (\%) \\
\hline Early & $2(4.9)$ \\
Seroma & 0 \\
Infection & $1(2.0)$ \\
Implant movement & 0 \\
Hematoma & $2(4.9)$ \\
Wound dehiscence & $1(2.0)$ \\
Red-breast syndrome & $1(2.0)$ \\
Implant loss & \\
Late & $2(4.9)$ \\
Rippling & $1(2.0)$ \\
Capsular contraction & \\
\hline
\end{tabular}




\section{Fig. 1. A case of prepectoral polyurethane breast reconstruction}

The patient underwent left skin-sparing mastectomy and left immediate prepectoral polyurethane implant reconstruction with Braxon matrix technique. (A) A preoperative photograph. (B) A postoperative photograph at 6 months follow-up. (C-E) A postoperative postradiotherapy photograph of left breast at 18 months follow-up and right risk-reducing mastectomy with polyurethane implant reconstruction with Braxon mesh technique.
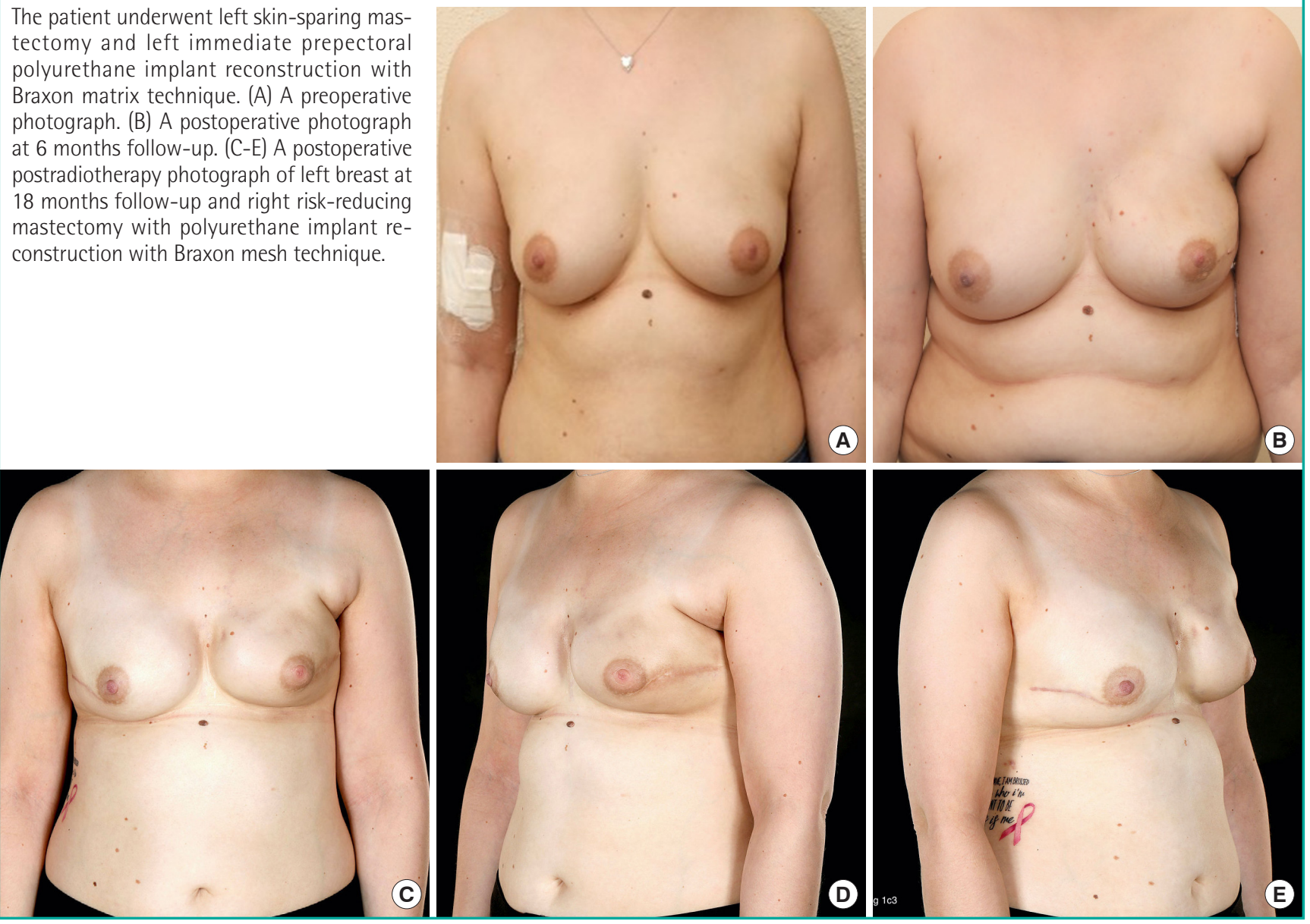

$\mathrm{mL}$ of fat was injected in the two patients who had lipomodelling. Postoperatively the uptake of the fat was good and patients had no complications. In our experience lipomodelling is a useful adjunct to correct rippling in prepectoral breast reconstruction.

An example of this reconstructive technique is shown in Fig. 1. A 31-year-old lady with body mass index (BMI) of $28 \mathrm{~kg} / \mathrm{m}^{2}$, had triple negative, medullary-like carcinoma on her left breast. She received neoadjuvant chemotherapy and underwent nipple sparing mastectomy weighing 495 g. Her breast was reconstructed with a $420 \mathrm{~mL}$ polyurethane implant and Braxon mesh technique. Postoperative adjuvant chemotherapy followed the surgery and seven months later, she agreed to have right breast risk-reducing mastectomy with similar to the contralateral breast reconstruction. She had no complications nor received lipomodelling.

\section{DISCUSSION}

Prepectoral implant-based breast reconstruction is on the rise due to advancements in implants and meshes. Overall our com- plications were lower than the recently published national iBRA (immediate breast reconstruction audit) [16]. We observed that patients with more than one comorbidity such as obese, diabetic, and smokers tend to develop more complications compared to women with no comorbidities. Moreover, appealing to note is that all the patients who presented complications had received at least two sessions of radiotherapy postoperatively. In contrast, patients who had received neither pre- nor postoperative radiotherapy did not develop any complications. Further studies would be advocated to provide further evidence for this.

In our series we observed only one implant loss (2\%) and this patient had comorbidities including obesity $\left(\mathrm{BMI}>35 \mathrm{~kg} / \mathrm{m}^{2}\right)$ and non-insulin dependent diabetes. The implant was salvaged using a tissue expander and changed to fixed volume implant after 6 months of postoperative radiotherapy. Majority of the patients who underwent prepectoral breast reconstruction (29\%) tolerated radiotherapy well although the follow up is short.

De Vita et al. [17] demonstrated data of 34 breast reconstruction with the use of polyurethane foam coated breast implants in the prepectoral position. Although the results of this study are 
preliminary, there were no major complications, nor grade IIIIV capsular contractions and excellent aesthetic outcome graded by BREAST-Q.

Moreover, studies have showed that complete ADM wrap reduces the incidence of capsular contracture by decreasing capsular fibrosis. In more details, since 1985 Ksander and Gray [18] described a reduced amount of capsule formation around the coated prostheses in rats while Komorowska-Timek et al. [19] studied the periprosthetic capsular contraction formation with AlloDerm. The latter study showed decreased radiation-related inflammation and delayed or diminished pseudoepithelium formation and thus contributed to slow progression of capsular formation, fibrosis, and contraction. Another experimental study by Schmitz et al. [20] supports the same hypothesis as the previous studies. In our series we observed that complete ADM wrap and the use of polyurethane implant had a good aesthetic outcome and only one patient of those who received a cycle of postoperative radiotherapy developed capsular contraction after 27 months of completion of radiotherapy and in total at 33 months of follow-up.

Further evidence for capsular contraction were given by a review by Headon et al. [21]. In this review, silicone implants and polyurethane were compared showing capsular contracture rates with silicone implants $10 \%$ to $15 \%$ at 6 years, whilst studies of polyurethane implants reported rates of $1.8 \%$ to $3.4 \%$. Yet, more research is required in this area for the results to be reliable. All eligible papers considered by the review stated positive outcomes for the use of polyurethane implants, especially for the reduction of capsular contracture. Another systematic review adds similar significant differences with the use of polyurethane implants compared to textured implants in developing capsular contracture [22].

In parallel, a study by Frame [23] supported the use of polyurethane implants without AMD mesh stating from his experience the rate of capsular contraction with such implants is less than $1 \%$ at 5 years and there were no cases of incidental breast cancer. This paper also outlined the other benefits of polyurethane implant, such as an alternative for mastopexy without concern of the implant weighing down the breast.

Another retrospective study by Pompei et al. [24] looked into the impact of radiotherapy on capsular contracture depending on the type of implant being used. Patient demographics, postoperative complications and cosmetic outcome were also recorded. Part of the study consisted of 166 patients and looked at two cohorts, patients who were given radiotherapy after breast reconstruction with implant expander and patients who did not receive radiotherapy. Braxon preshaped mesh ex vivo was not used in this study. In each cohort the type of implant was also stated; this was between either a textured implant or a polyurethane implant. The results suggested that the use of polyurethane implants following postmastectomy radiotherapy reduced capsular contracture rate by $15.4 \%$ from $21.7 \%$ to $6.3 \%$ compared to the textured implant in the setting of an immediate delayed breast reconstruction. No capsular contracture was recorded in patients without radiotherapy where implant-based breast reconstruction utilized polyurethane implants, but a rate of $8.3 \%$ was seen in patients with textured implants for capsular contracture.

Ultimately, besides the low rate in capsular contraction, it has been previously observed by the senior author that the use of non-polyurethane implant had a higher rate of rippling [25]. In our study, only two patients presented with breast rippling. Each of those patients received one to two sessions of lipomodelling with satisfactory cosmetic results. Unfortunately, in our study, we did not include patients' satisfaction assessment, a limiting factor which we do recognize and plan to address in the future.

Prepectoral implant-based reconstruction appears to be a useful tool in oncoplastic breast reconstruction. To conclude, we believe this is one of the few papers to report on the outcome of the prepectoral use of complete $\mathrm{ADM}$ wrap using polyurethane in immediate implant-based breast reconstruction. Our early observational series show satisfactory outcome and this technique appears to be promising.

\section{NOTES}

\section{Conflict of interest}

No potential conflict of interest relevant to this article was reported.

\section{Ethical approval}

The study was approved by the Institutional Review Board of The Royal of Wolverhampton NHS Trust (audit No. 4135) and performed in accordance with the principles of the Declaration of Helsinki. Written informed consents were obtained.

\section{Patient consent}

The patient provided written informed consent for the publication and the use of her images.

\section{Author contribution}

Conceptualization: F Neamonitou. Data curation: F Neamonitou, F Salem. Formal analysis: F Neamonitou. Methodology: F Neamonitou. Project administration: F Neamonitou. Writing original draft: F Neamonitou. Writing - review \& editing: F Neamonitou, S Mylvaganam, R Vidya. 


\section{ORCID}

Foteini Neamonitou https://orcid.org/0000-0003-2432-0917 Senthurun Mylvaganam

https://orcid.org/0000-0002-0059-2275

Fathi Salem https://orcid.org/0000-0001-6308-414X

Raghavan Vidya ～https://orcid.org/0000-0002-6129-2444

\section{REFERENCES}

1. Vidya R, Iqbal FM. A guide to prepectoral breast reconstruction: a new dimension to implant-based breast reconstruction. Clin Breast Cancer 2017;17:266-71.

2. Oliver JD, Boczar D, Huayllani MT, et al. Postmastectomy radiation therapy (PMRT) before and after 2-stage expander-implant breast reconstruction: a systematic review. Medicina (Kaunas) 2019;55:226.

3. Elswick SM, Harless CA, Bishop SN, et al. Prepectoral implant-based breast reconstruction with postmastectomy radiation therapy. Plast Reconstr Surg 2018;142:1-12.

4. Castel N, Soon-Sutton T, Deptula P, et al. Polyurethanecoated breast implants revisited: a 30-year follow-up. Arch Plast Surg 2015;42:186-93.

5. Van Zele D, Heymans O. Breast implants: a review. Acta Chir Belg 2004;104:158-65.

6. Medtech Insight. Bristol-Myers Squibb polyurethane-coated breast implant study [Internet]. Tustin, CA: Medtech Insight; c1993 [cited 2019 Aug 15]. Available from: https:// medtech.pharmaintelligence.informa.com/MT001252/ BRISTOLMYERS-SQUIBB-POLYURETHANECOATED-BREAST-IMPLANT-STUDY.

7. Spear SL, Parikh PM, Goldstein JA. History of breast implants and the food and drug administration. Clin Plast Surg 2009;36:15-21.

8. Reitsamer R, Peintinger F. Prepectoral implant placement and complete coverage with porcine acellular dermal matrix: a new technique for direct-to-implant breast reconstruction after nipple-sparing mastectomy. J Plast Reconstr Aesthet Surg 2015;68:162-7.

9. Vidya R, Masia J, Cawthorn S, et al Evaluation of the effectiveness of the prepectoral breast reconstruction with Braxon dermal matrix: first multicenter European report on 100 cases. Breast J 2017;23:670-6.

10. Robertson SA, Rusby JE, Cutress RI. Determinants of optimal mastectomy skin flap thickness. Br J Surg 2014;101: 899-911.

11. Liliav B, Patel P, Jacobson AK. Prepectoral breast reconstruction: a technical algorithm. Plast Reconstr Surg Glob Open 2019; 7:e2107.
12. Abdelhamid MI, Alkilany MM, Lotfy M. Pectoral fascia preservation during modified radical mastectomy: why and when. Egypt J Surg 2017;36:333-5.

13. Caputo GG, Marchetti A, Dalla Pozza E, et al. Skin-reduction breast reconstructions with prepectoral implant. Plast Reconstr Surg 2016;137:1702-5.

14. Thuman J, Freitas AM, Schaeffer C, et al. Prepectoral wisepattern staged implant-based breast reconstruction for obese or ptotic patients. Ann Plast Surg 2019;82(6S Suppl 5):S404-9.

15. Vidya R, Berna G, Sbitany H, et al. Prepectoral implantbased breast reconstruction: a joint consensus guide from UK, European and USA breast and plastic reconstructive surgeons. Ecancermedicalscience 2019;13:927.

16. Potter S, Conroy EJ, Cutress RI, et al. Short-term safety outcomes of mastectomy and immediate implant-based breast reconstruction with and without mesh (iBRA): a multicentre, prospective cohort study. Lancet Oncol 2019;20:25466.

17. de Vita R, Buccheri EM, Villanucci A, et al. Breast reconstruction actualized in nipple-sparing mastectomy and direct-to-implant, prepectoral polyurethane positioning: early experience and preliminary results. Clin Breast Cancer 2019;19:e358-63.

18. Ksander GA, Gray L. Reduced capsule formation around soft silicone rubber prostheses coated with solid collagen. Ann Plast Surg 1985; 14:351-60.

19. Komorowska-Timek E, Oberg KC, Timek TA, et al. The effect of AlloDerm envelopes on periprosthetic capsule formation with and without radiation. Plast Reconstr Surg 2009; 123:807-16.

20. Schmitz M, Bertram M, Kneser U, et al. Experimental total wrapping of breast implants with acellular dermal matrix: a preventive tool against capsular contracture in breast surgery? J Plast Reconstr Aesthet Surg 2013;66:1382-9.

21. Headon H, Kasem A, Mokbel K. Capsular contracture after breast augmentation: an update for clinical practice. Arch Plast Surg 2015;42:532-43.

22. Duxbury PJ, Harvey JR. Systematic review of the effectiveness of polyurethane-coated compared with textured silicone implants in breast surgery. J Plast Reconstr Aesthet Surg 2016;69:452-60.

23. Frame JD. The arguments for polyurethane covered breast implants in cosmetic and reconstructive breast surgery. PMFA News 2014;1:20-2.

24. Pompei S, Arelli F, Labardi L, et al. Breast reconstruction with polyurethane implants: preliminary report. Eur J Plast Surg 2011;35:441-7. 
25. Vidya $\mathrm{R}$, Iqbal FM, Becker $\mathrm{H}$, et al. Rippling associated with grading system. World J Plast Surg 2019;8:311-5. pre-pectoral implant based breast reconstruction: a new 